\title{
Tests of gas contaminants and interaction with materials in RPC closed loop recirculation systems
}

\author{
R. Aurilio ${ }^{a}$, L. Benussi ${ }^{a}$, S. Bianco ${ }^{a}$, S. Colafranceschi ${ }^{b}$, M. Ferrini $^{c}$, T. Greci ${ }^{b}$, \\ L. Passamonti ${ }^{a}$, D. Piccolo ${ }^{a}$, D. Pierluigi ${ }^{a}$, A. Russo ${ }^{a}$, G. Saviano ${ }^{c}$, C. Vendittozzi ${ }^{c}$ \\ ${ }^{a}$ Laboratori Nazionali Frascati (INFN). \\ ${ }^{b}$ CERN, Laboratori Nazionali Frascati (INFN) and Università degli studi di Roma - La Sapienza. \\ ${ }^{c}$ Laboratori Nazionali Frascati (INFN) and Università degli studi di Roma - La Sapienza.
}

E-mail: stefano.colafranceschiecern.ch

Resistive Plate Counters (RPC) detectors at the Large Hadron Collider (LHC) experiments normally use gas recirculation systems to cope with large gas mixture volumes and costs. A new long-term systematic study of gas purifiers has been carried out at CERN in a low-radiation test area, with the use of RPC chambers with currents monitoring and gas analysis. The study aims at measuring the lifetime of purifiers, release of contaminants, and to characterize the behavior of RPC dark currents as a function of contaminants.

XI Workshop on Resistive Plate Chambers and Related Detectors (RPC2012)

5-10 February, 2012

Frascati, Italy.

${ }^{*}$ Corresponding author 


\section{Introduction}

Resistive Plate Counters[四] (RPC) detectors are installed at both the ATLAS (A Toroidal LHC Apparatus)[[] and CMS (Compact Muon Solenoid)[B] experiments at the LHC (Large Hadron Collider) of CERN, Geneva (Switzerland) to provide triggering and synchronization in both barrel and endcaps regions as part of the muon system. RPCs use a freon-based gas mixture (typically $95.2 \% \mathrm{C}_{2} \mathrm{H}_{2} \mathrm{~F}_{4}-4.5 \%$ Iso- $\mathrm{C}_{4} \mathrm{H}_{10}-0.3 \% \mathrm{SF}_{6}$ humidified at the $40 \% \mathrm{RH}$ ) in a recirculation system call Closed Loop (CL) [四].

The CL was designed to cope with large gas mixture volumes and costs. In the closed loop system industrial filters commercially available are in operation to purify the mixture and to prevent contamination collection that affects the RPC performances.

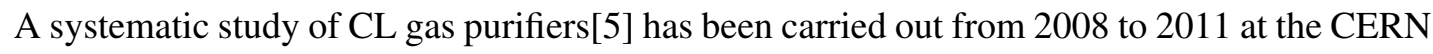
using RPC chambers exposed to cosmic rays and a scaled-down closed loop gas system equipped with several gas analysis sampling points. Goals of the study[焑] were to observe the release of contaminants in correlation with the dark current increase in RPC detectors, to measure the purifier lifetime $[\square]$ with unused material, to observe the presence of pollutants. In this paper, new preliminary results from the 2011 run, characterize the behavior of used purifiers, and study the pattern of dark currents increase in the upstream versus the downstream gaps.

\section{Experimental setup}

The experimental setup [ $[\mathbb{[}][0]$ is composed of a closed and an open loop gas systems (Fig. W). To purify the closed loop gas mixture (that continuously receives $10 \%$ of fresh mixture), commercial filters are adopted as shown in Fig. $\square$.

The first purifier consists in a $5 \AA \quad(10 \%)$ and $3 \AA \quad(90 \%)$ type zeolite molecular sieve (ZEOCHEM[ए]]). The second cartridge is filled with $50 \% \mathrm{Cu}-\mathrm{Zn}$ filter type R12 (BASF[प]]) and $50 \% \mathrm{Cu}$ filter type $\mathrm{R} 3-11 \mathrm{G}$ (BASF) while the third consists of $\mathrm{NiAlO}_{3}$ filter type 6525 (LEUNA[ए2]).

Eleven double-gap RPC detectors are installed in a temperature and humidity controlled hut, with online monitoring of environmental parameters, nine out of eleven are operated in closed loop while two in open mode. During the data-taking the number of double-gap detectors decreased from 9 to 7 due to the fact that 2 RPC becomed not operational anymore. Each RPC detector has two gaps (upstream and downstream) whose gas lines are serially connected. The detectors are operated at a $9.2 \mathrm{kV}$, at this working point the anode dark current drawn (due to the high bakelite resistivity) is approximately 1-2 $\mu \mathrm{A}$. Gas sampling points, before and after each filter of the closed loop, allows chemical and gaschromatograph analysis[[]]. Gas mixture composition is monitored twice a day by gaschromatograph, which also provides the amount of air contamination, stable over the entire data-taking run and below 300 (100) ppm in closed (open) loop as in Fig. [1].

\section{Chemical analysis setup}

Chemical analyses have been performed in order to study the dynamical behavior of dark currents increase of the RPC detectors. To identify the contaminants nature, the gas was sampled

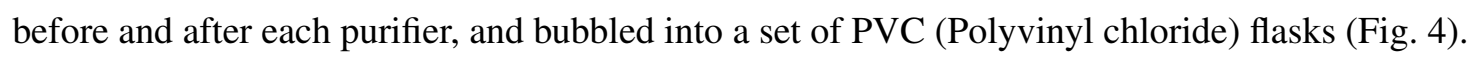




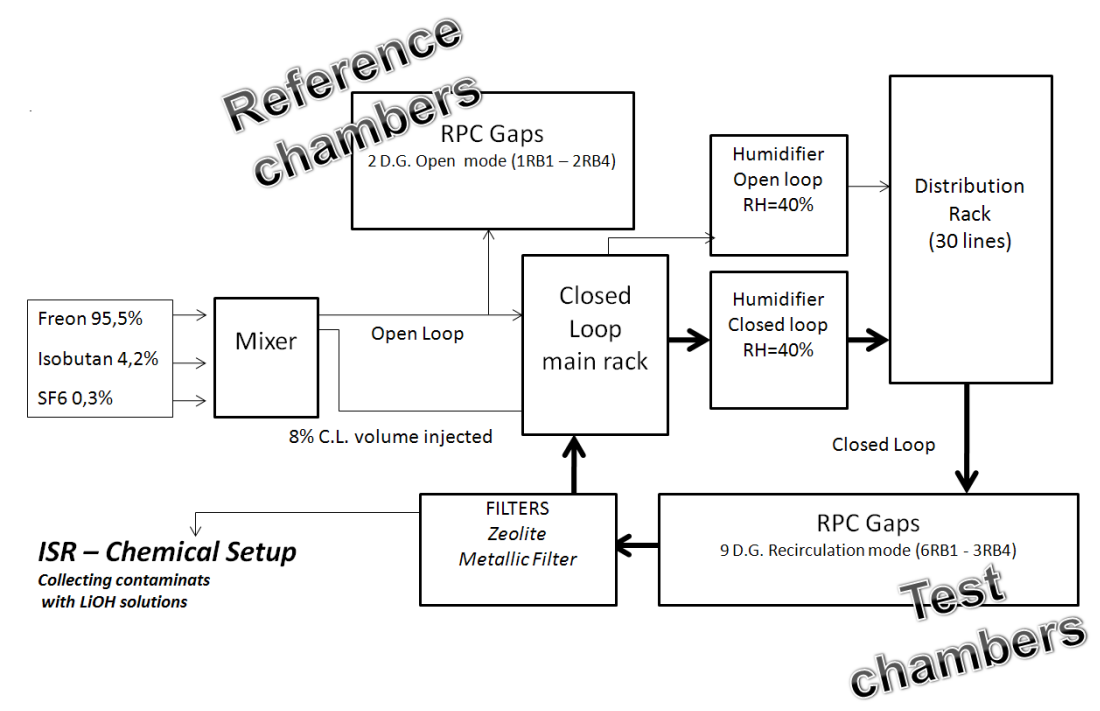

Figure 1: Schema of the CL setup.

Flask 1 acted as a buffer to avoid return of LiOH into the CL. Flasks 2, 3 and 4 contained $250 \mathrm{ml}$ solution of $\mathrm{LiOH}(0.001 \mathrm{~mol} / \mathrm{l}$ corresponding to $0.024 \mathrm{~g} / \mathrm{l}$, optimized to keep the $\mathrm{pH}$ of the solution at 11). The bubbling of gas mixture into the three flasks allows to capture a wide range of elements that are likely to be released by the system, such as $\mathrm{Ca}, \mathrm{Na}, \mathrm{K}, \mathrm{Cu}, \mathrm{Zn}, \mathrm{Ni}, \mathrm{F}$. At the end of each sampling line the flow is measured to estimate the total amount of gas for the whole period of data-taking. Sampling points HV61,64 (Fig. (⿴) are located before filters (HV61) after zeolite (HV62), after Cu/Zn filter (HV64), after Ni filter (HV66).

To measure the fluorine production the sampling point HV61 and HV62 were equipped with two additional flaks and fluoride selective electrodes. The $\mathrm{F}^{-}$selective electrode adopted[?] was a solid state half-cell sensor that requires, as separate referencea silver-silver chloride double junction half-cell reference electrode. Selective electrodes were installed to measure the ionic potential, which is directly connected to the ions concentration.

The selective electrodes were monitoring the actual collected amount of ions integrating it over time, thanks to a custom $a d$ - hoc software the electrochemical potential was logged every 10 minutes in order to estimate the $\mathrm{F}^{-}$production rate and concentration in the system along with the RPC performance. Both electrodes (reference and sensor) were immersed in a diluted TISAB II solution. This increases and stabilizes the ionic strength of the solution making a linear correlation between the logarithm of the concentration of analyte. The calibration of the two selective electrodes was performed at the beginning of the run and also during the run itself to double-check a possible shift of the factory settings. For the calibration we have used known solutions containing: $0.001 \mathrm{mg} / \mathrm{l}$, $0.005 \mathrm{mg} / 1,0.01 \mathrm{mg} / 1,0.10 \mathrm{mg} / 1,1.0 \mathrm{mg} / \mathrm{l}, 10.0 \mathrm{mg} / \mathrm{l}, 20.0 \mathrm{mg} / 1,50.0 \mathrm{mg} / \mathrm{l}$ concentration of $\mathrm{F}^{-}$; Fig. 1 l shows the calibration curves. 


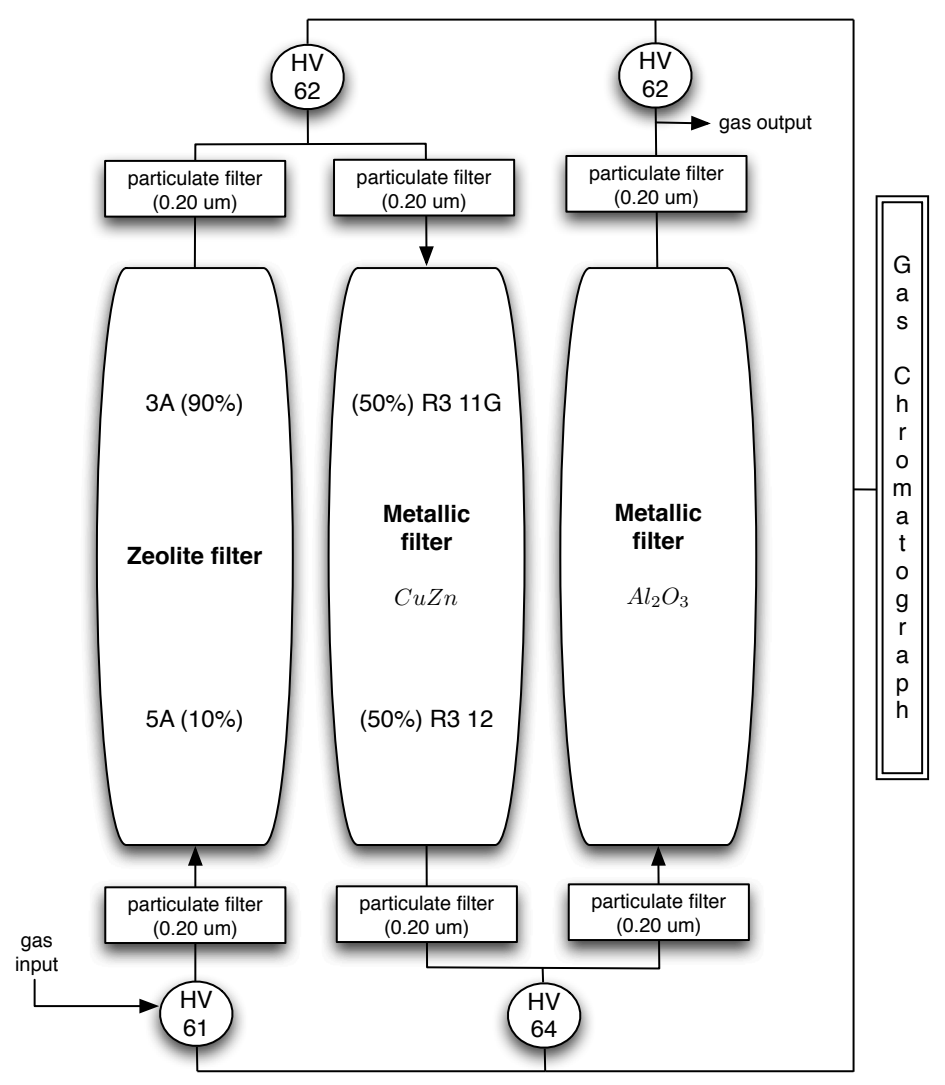

Figure 2: Purifiers equipped with several sampling points (HV61, 62, 64) before and after each stage.

\section{Results}

To mark the different operating conditions (Tab. W), the data-taking period was divided into two runs over three years.

Each run is characterized by cycles of operation. Fig. $\square$ shows the average of all RPC anodic dark currents $I_{i}(t)$ over $n$ gaps, normalized by their initial values $I_{i}\left(t_{0}\right)$. The z-axis scale (colorcoded) shows the $\mathrm{F}^{-}$produced by the system. The increase of dark anode currents of up-stream gaps in run 1 and run 2 is clearly visible, as well as the increase of $\mathrm{F}^{-}$concentration. The dark currents of down-stream gaps, as well as the currents of all RPCs in OL, are found stable.

In run 1, the purifier cartridges are filled with unused material. Twenty-two RPC detectors where used. Cycle 1 and cycle 2 have stable currents up to April 2009, when an increase in the dark current occurs for all up-stream gaps in CL, leaving the down-stream gaps stable. Cycle 4 in particular shows a clear increase of currents, and was terminated before permanent damage occurred to the detector. The lifetime of purifiers is determined by evaluating the duration of cycle 1 and cycle 2 :

$$
\tau_{\text {run } 1}=211 \pm 2 \text { days }
$$

The total gas flow is $63 \pm 3 \mathrm{l} / \mathrm{h}$. The fluorine production of RPCs in closed loop was measured 


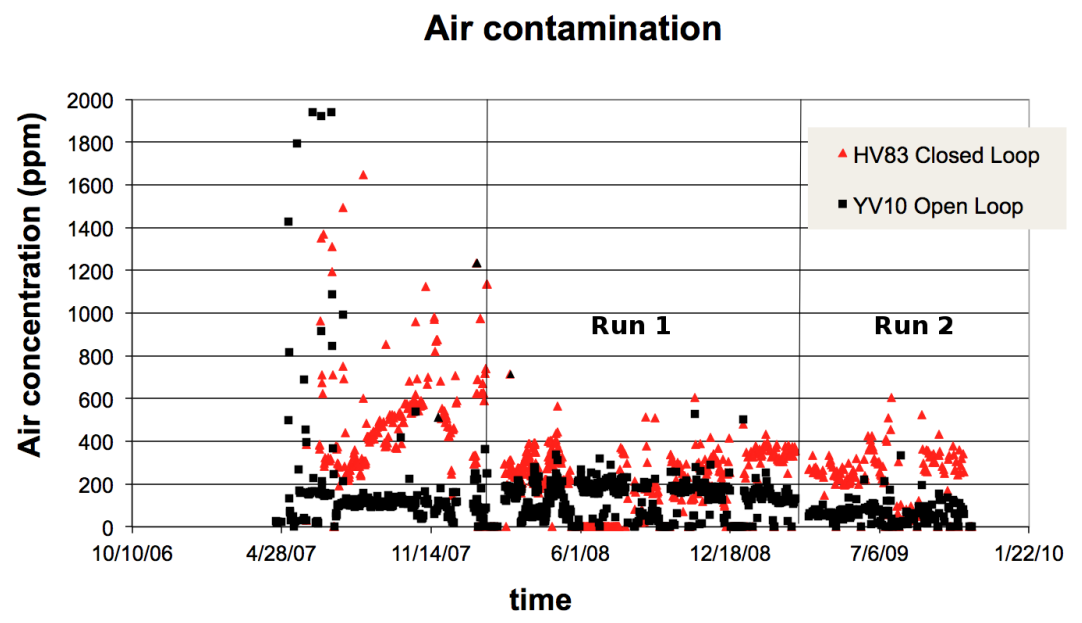

Figure 3: Air contamination measured in the open and closed loop recirculation system.

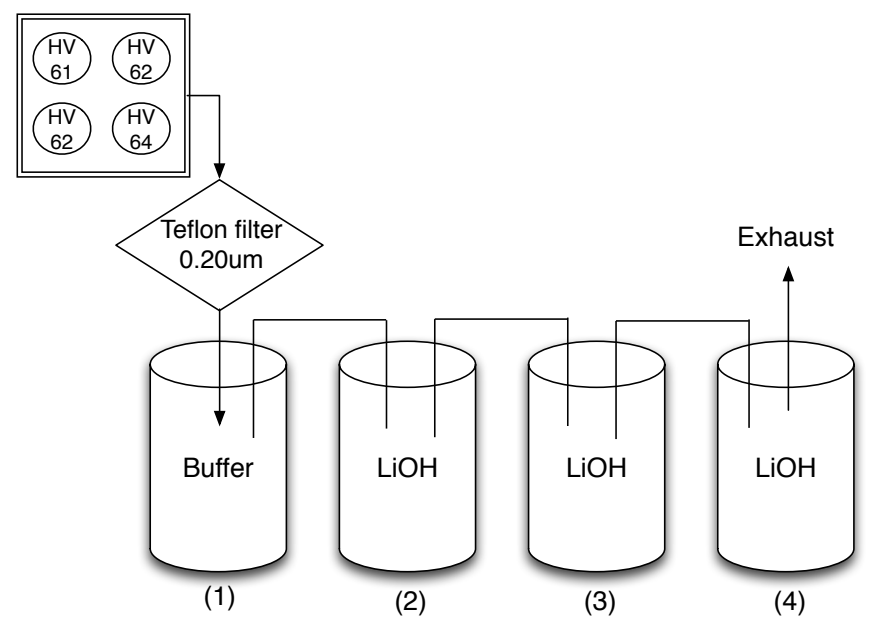

Figure 4: Chemical analyses are performed using LiOH flasks in which gas is bubbled and contaminants collected.

previously in high-radiation condition [[13], [14]]. In our study we measure the fluorine production (Fig. Q) during run 1 as $1.10 \pm 0.05 \mu \mathrm{mol} / 1$ corresponding to a total accumulation in the CL of $(45 \pm$ 2) $10^{3} \mu \mathrm{mol} / 1$. Fig. $\$$ shows the typical behaviour of one RPC detector in closed loop correlated with the concentration of the main contaminants found.

Before starting run 2, all purifiers are regenerated following the CERN gas group standard procedure, i.e. by means of a flushing of hot $\left(215^{\circ} \mathrm{C}\right) \mathrm{Ar}$ and $\mathrm{H}_{2}$ mixture (80:20) for twelve hours. During run 2 the flux is measured $\approx 54 \pm 31 / \mathrm{h}$. Twenty RPC detectors were used. The currents of down-stream gaps are found stable throughout all cycles as in run 1, while the currents of the up-stream gaps increase. As a cross-check, gas supplies of two gaps of the same RPC detector were swapped to check that in a pair of gaps only the up-stream gap showed currents increase. The 


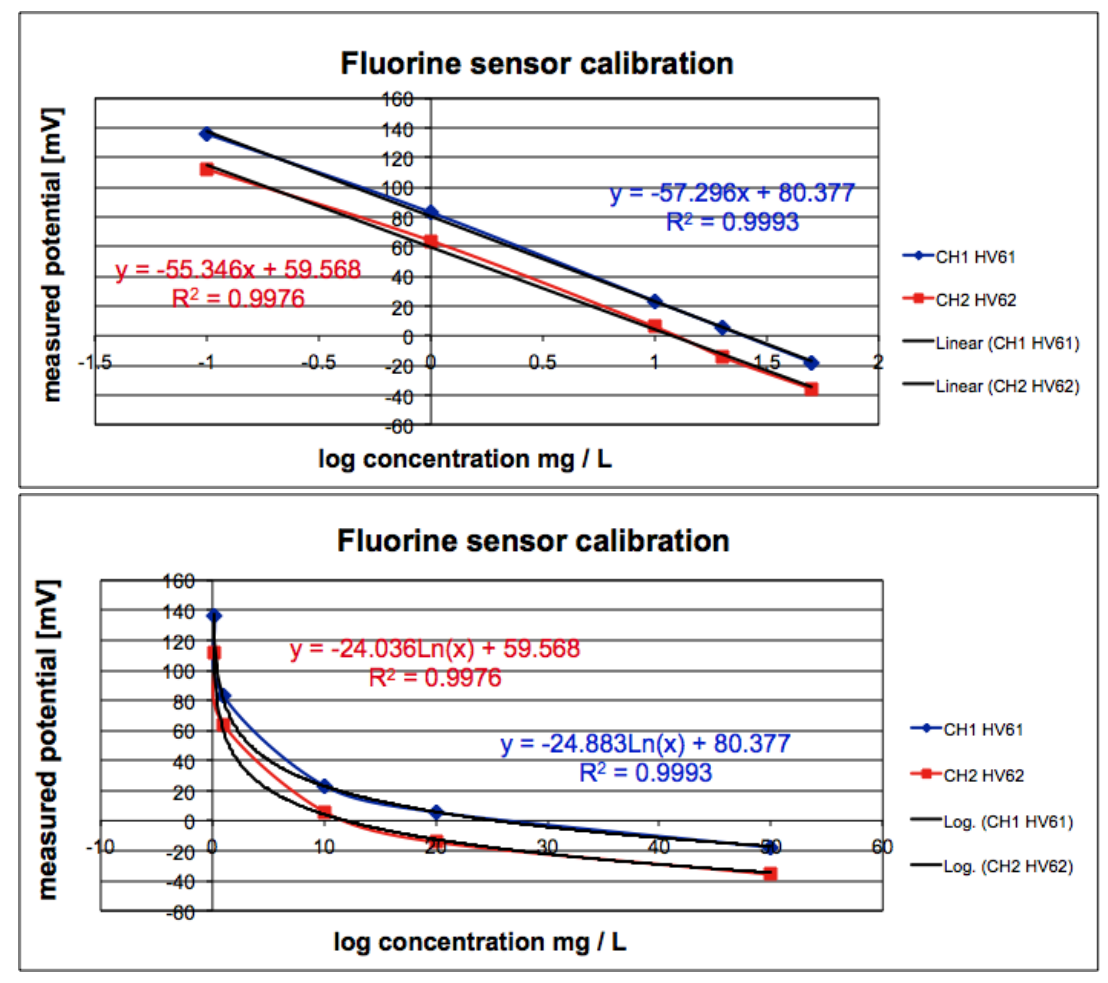

Figure 5: Calibration curves of fluorine monitoring sensors.

Table 1: Summary table of Closed Loop (CL) and Open Loop (OL) channels.

\begin{tabular}{|c|c|c|ccc|}
\hline Run & Cycle & Period & \multicolumn{3}{|c|}{ Comment } \\
\hline 1 & 1 & $29 / 08 / 2008-11 / 10 / 2008$ & stable currents & CL & unused filters \\
1 & 2 & $12 / 10 / 2008-22 / 01 / 2009$ & stable currents & CL & unused filters \\
1 & 3 & $23 / 01 / 2009-28 / 04 / 2009$ & increasing currents & CL & unused filters \\
1 & 4 & $29 / 04 / 2009-14 / 07 / 2009$ & increasing currents & CL & unused filters \\
1 & 5 & $15 / 07 / 2009-27 / 07 / 2010$ & decreasing current & OL & used filters \\
2 & 1 & $28 / 07 / 2010-07 / 01 / 2011$ & stable currents & CL & used filters \\
2 & 2 & $08 / 01 / 2011-05 / 07 / 2011$ & increasing currents & CL & used filters \\
\hline
\end{tabular}

lifetime of regenerated purifiers is evaluated:

$$
\tau_{\text {run } 2}=160 \pm 2 \text { days }
$$

The $\mathrm{F}^{-}$production is measured $0.84 \pm 0.05 \mu \mathrm{mol} / 1 \mathrm{Fig}$. Q , corresponding to an accumulation of $33 \pm 2) 10^{3} \mu \mathrm{mol} / 1$. Analyses are in progress in order to confirm the release of contaminants observed in run 1 and shown in Fig. 8 .

The lesser $\mathrm{F}^{-}$production in run 2 with respect to run 1 is interpreted as due to the smaller number of detectors used in run 2. Although the lifetime of purifiers is measured different in run 


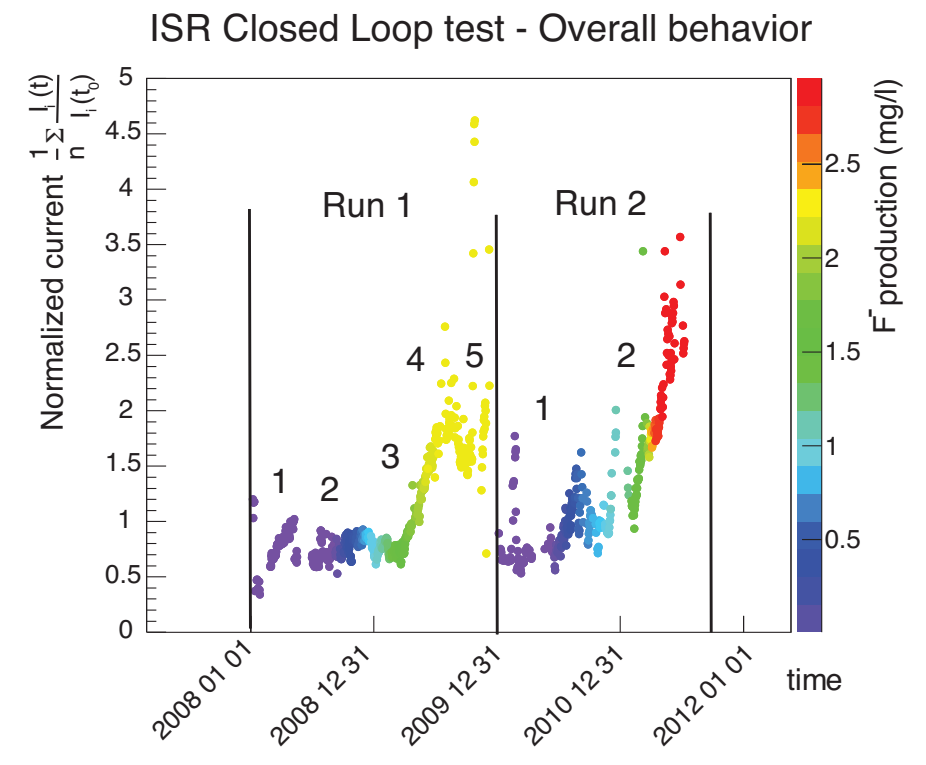

Figure 6: Upstream gap total current during all runs with $\mathrm{F}^{-}$production.

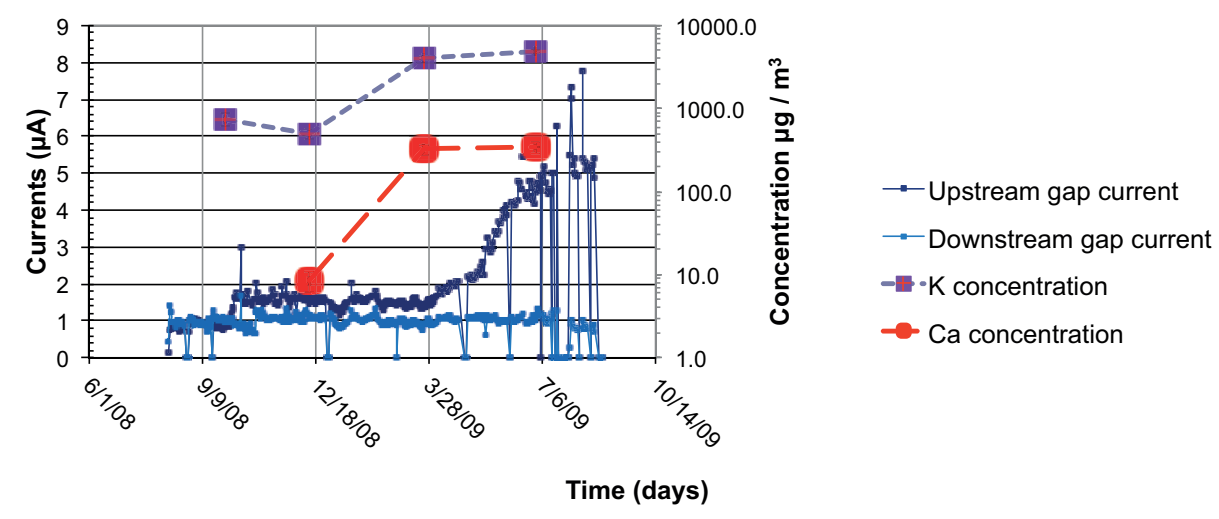

Figure 7: Dark currents increase (run 1) in the up-stream gap and not in the down-stream gap, correlated to the detection of gas contaminants measured using the chemical analysis setup.

1 and run 2, the lifetime normalized by the $\mathrm{F}^{-}$production is found compatible within errors, i.e., $4.64 \pm 0.24$ days $/ \mathrm{mmol} / 1$ for run 1 and $4.68 \pm 0.25$ days $/ \mathrm{mmol} / \mathrm{l}$ for run 2 .

During both run 1 and run 2 the production of $\mathrm{F}^{-}$is efficiently depressed by the Zeolite purifier as shown in Fig. $Q$. The presence of an excess production of $\mathrm{K}$ and $\mathrm{Ca}$ in coincidence with the currents increase also suggests a damaging effect of HF[ [प]], produced in the system, on the zeolite framework of that is based on $\mathrm{K}$ and $\mathrm{Ca}$. Further analysis are ongoing to identify the contaminants present during run 2. 


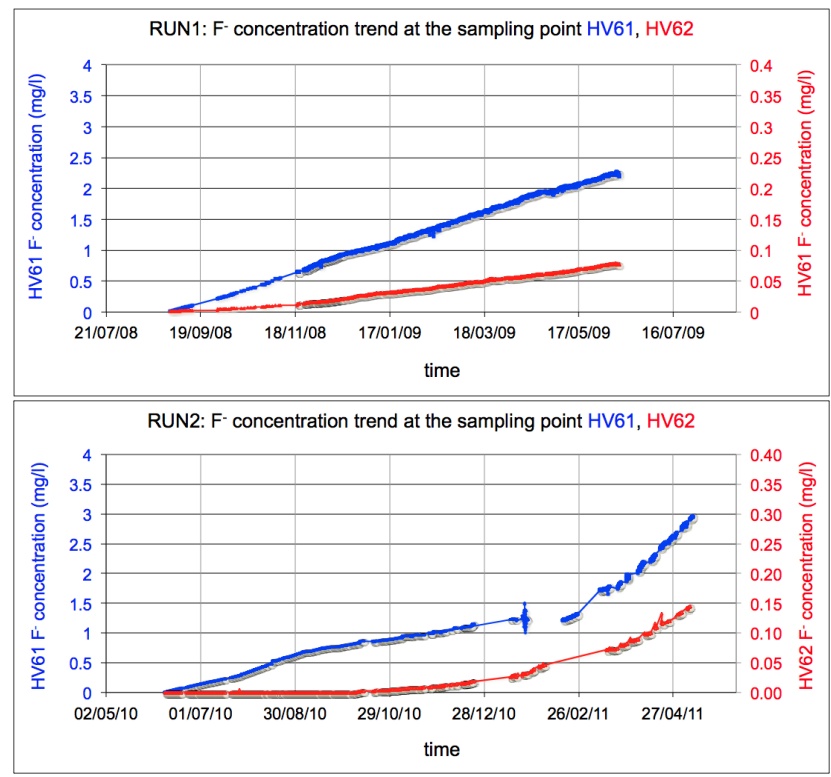

Figure 8: $\mathrm{F}^{-}$production during run 1 (unused filters) and run 2 (used filters).

\section{Conclusions}

In this paper preliminary results on studies of gas contaminants and characterizations of RPC performance along with different conditions of the CL gas system of the CMS RPC muon detector were reported. Chemical analysis were performed by using gaschromatography, $\mathrm{pH}$ sensors and contaminants detectors. Lifetime of unused purifiers is more than 6 months, after that the RPC current of up-stream gap start increasing, while the down-stream gaps show stable currents. This suggests that the first gap acts as a mechanical filter to the second gap which does not receive a polluted gas mixture. During the test run with regenerated purifiers, after 5 months, the $\mathrm{F}^{-}$ production rate increased as well as the up-stream current confirming the effect seen during the run with unused materials.

The $\mathrm{F}^{-}$production during run 2 was $25 \%$ smaller if compared to the $\mathrm{F}^{-}$production occurred during run 1. This is justified by the fact that the number of powered RPC changed due to broken gaps, while during run 1 the current averaged among all gaps was $2.29 \mu \mathrm{A}$. in the run 2 the average current decreased to $1.70 \mu \mathrm{A}$., with a reduction of $26 \%$.

Run 1 proved to run with unused filters for $211 \pm 2$ days, run 2 lasted $160 \pm 2$ days with used filters. While during run 2 the $\mathrm{F}^{-}$production was $\approx 25 \%$ smaller, the purifier lifetime, normalized with $\mathrm{F}^{-}$production was found to be $4.64 \pm 0.24$ days $/ \mathrm{mmol} / \mathrm{l}$ during run 1 and $4.68 \pm 0.25$ days $/ \mathrm{mmol} / 1$ in run 2.

\section{References}

[1] R. Santonico and R. Cardarelli, “Development Of Resistive Plate Counters,” Nucl. Instrum. Meth. 187 (1981) 377 . 
[2] The ATLAS Collaboration et al., "The ATLAS Experiment at the CERN Large Hadron Collider" 2008 JINST 3 S08003.

[3] S. Chatrchyan et al. [CMS Collaboration], "The CMS experiment at the CERN LHC," JINST 3 (2008) S08004.

[4] M. Bosteels et al., “CMS Gas System Proposal”, CMS Note 1999/018. L. Besset, F. Hahn, S. Haider, C. Zinoni, Experimental Tests with a Standard Closed Loop Gas Circulation System, CMS Note 2000/040.

[5] G. Saviano et al., "Materials studies for the RPC detector in CMS ", presented at the RPC07 Conference, Mumbai (India), January 2008.

[6] M. Abbrescia et al., "Proposal for a Systematic Study of the CERN Closed Loop Gas System Used by the RPC Muon Detectors in CMS",

[7] L. Benussi, S. Bianco, S. Colafranceschi, F. L. Fabbri, F. Felli, M. Ferrini, M. Giardoni and T. Greci et al., "Study of gas purifiers for the CMS RPC detector," Nucl. Instrum. Meth. A 661 (2012) S241 [arXiv:1012.5511 [physics.ins-det]].

[8] S. Bianco, S. Colafranceschi, D. Colonna, F. Felli, T. Greci, A. Paolozzi, L. Passamonti and D. Pierluigi et al., "Chemical Analyses Of Materials Used In The Cms Rpc Muon Detector," CERN-CMS-NOTE-2010-006.

[9] S. Colafranceschi, "A study of materials used for muon chambers at the CMS Experiment at the LHC: interaction with gas, new materials and new technologies for detector upgrade", Ph.D. thesis.

[10] Manufactured by ZEOCHEM AG, Seestrasse 108, 8708 Uetikon (Germany).

[11] BASF Technical Bulletin.

[12] LEUNA Data Sheet September 9, 2003, Catalyst KL6526-T.

[13] G. Aielli et al., "Fluoride production in RPCs operated with F-compound gases", 8th Workshop on Resistive Plate Chambers and Related Detectors, Seoul, Korea, 10-12 Oct 2005. Published in Nucl.Phys.Proc.Suppl. 158 143-148, 2006.

[14] M. Abbrescia et al., "HF Production In Cms-Resistive Plate Chambers,” Nucl. Phys. Proc. Suppl. 158 (2006) 30. NUPHZ,158,30; 